\title{
CACERÍA DE MAMÍFEROS MEDIANOS EN CAFETALES DEL CENTRO DE VERACRUZ, MÉXICO
}

\author{
Liliana TlaPaya ${ }^{1}$ y Sonia Gallina ${ }^{2}$ \\ ${ }^{1}$ Escuela de Biología, Benemérita Universidad Autónoma de Puebla. Boulevard Valsequillo \\ y Av. San Claudio Edificio 76, Ciudad Universitaria, Col. Jardines de San Manuel C. P. 72570, \\ Puebla, Puebla, MÉXICO. \\ liliana_tlapaya@yahoo.com.mx \\ 2 Red Biología y Conservación de Vertebrados. Instituto de Ecología, A. C. Carretera Antigua \\ a Coatepec No. 351, El Haya, CP 91070, Xalapa, Veracruz, MÉXICO. \\ sonia.gallina@inecol.edu.mx
}

Tlapaya, L. \& S. Gallina. 2010. Cacería de mamíferos medianos en cafetales del centro de Veracruz, México. Acta Zool. Mex. (n. s.), 26(2): 259-277.

RESUMEN. El presente trabajo analiza la cacería de mamíferos medianos en cafetales del centro de Veracruz, México, enfocado en dos zonas: Huatusco y Teocelo-Coatepec, registrando datos por medio de entrevistas estructuradas aplicadas a cazadores activos en las zonas de estudio. Los cafetales son agroecosistemas que presentan un sistema de manejo bajo sombra de árboles, que permiten mantener una biodiversidad considerable de mamíferos medianos. La colecta de datos se realizó durante los meses de enero a mayo del 2006 en los cafetales muestreados como parte de un proyecto más amplio titulado BIOCAFE. Se aplicaron un total de 77 entrevistas estructuradas que nos permitieron conocer qué mamíferos son cazados, los métodos utilizados para cazarlos, el uso que les dan, además de inferir cuantos animales se extraen por año y que biomasa representa. Los resultados de las entrevistas muestran que de las 24 especies registradas de mamíferos medianos, son cinco especies las más cazadas en ambas zonas en proporciones significativas: armadillo (Dasypus novemcinctus), tlacuache (Didelphis virginiana), conejo (Sylvilagus floridanus), mapache (Procyon lotor) y zorra (Urocyon cinereoargenteus). De las 16 especies cazadas, el armadillo es la presa de mayor demanda y la más aprovechada en ambas zonas. La estimación de biomasa extraída al año fue de $194 \mathrm{~kg} /$ cazador para la zona de Teocelo-Coatepec y de $174 \mathrm{~kg} /$ cazador en la zona Huatusco. En cuanto a los métodos más comúnmente utilizados para cazar son; arma de fuego (53\%), seguido del uso de perros (41\%) y por último la arreada (33\%). La presión de cacería fue mucho mayor en los cafetales de la zona de Teocelo-Coatepec por haber una mayor densidad poblacional humana. La cacería que se practica en ambas zonas es completamente ilegal, es por esto que es necesario realizar acciones para promover el manejo y la conservación de las especies, así como al desarrollo de la comunidad en el área de estudio.

Palabras clave: Biodiversidad, biomasa extraída, cacería, entrevistas estructuradas, Huatusco, TeoceloCoatepec, Veracruz.

Recibido: 15/04/2009; aceptado: 19/02/2010. 
Tlapaya, L. \& S. Gallina. 2010. Hunting of medium mammals in coffee plantations in central Veracruz, Mexico. Acta Zool. Mex. (n. s.), 26(2): 259-277.

ABSTRACT. This study analyzes the hunting of medium mammals in coffee plantations in central Veracruz, México, focusing on two areas: Huatusco and Teocelo-Coatepec. The data were obtained through a guided interview, which was applied to active hunters in the study areas. The data collection was done during January to May 2006 as part of a larger project entitled BIOCAFE. We applied a total of 77 interviews that allowed us to know the mammals hunted, the methods used, how they used them, in addition to infer how many animals are extracted per year and the biomass that represented. The records showed that five species were mainly hunted in both areas: armadillo (Dasypus novemcinctus), opossum (Didelphis virginiana), rabbit (Sylvilagus floridanus), raccoon (Procyon lotor) and gray fox (Urocyon cinereoargenteus). Of the 16 hunted species, the armadillo is the most common prey and the most used in both areas. The estimated extracted biomass per year was $194 \mathrm{~kg} / \mathrm{hunter}$ in the zone of Teocelo-Coatepec and $174 \mathrm{~kg} / \mathrm{hunter}$ in the Huatusco zone. The most common hunting methods are: by fire arm $(53 \%)$, followed by the use of dogs $(41 \%)$ and last, the beat $(33 \%)$. The hunting pressure was greater in coffee plantations in the zone of Teocelo-Coatepec for having a higher human population density. Hunting practiced in both areas is completely illegal, hence is necessary to apply actions to promote the management and conservation of species, together with community development in the study area.

Keywords: Biodiversity, biomass extracted, hunting, interviews, Huatusco, TeoceloCoatepec, Veracruz.

\section{INTRODUCCIÓN}

Las especies a lo largo del tiempo han interactuado necesariamente unas con otras de distintos modos, y el hombre ha ejercido una presión sobre ellas de tal forma que el impacto humano sobre la extinción de las especies abarca un periodo de varios milenios a partir de la prehistoria con una aceleración de la pérdida de especies durante los últimos 500 años (Reid \& Miller 1989). La transformación de los sistemas naturales directamente relacionada con las actividades humanas es un factor determinante, por lo que un número de especies han visto disminuir rápidamente sus poblaciones, a tal grado que algunas están al borde de la extinción, por diversas causas como la caza irracional, la destrucción de hábitats y por efecto de la competencia y depredadores introducidos (Heywood 1995, Lawton \& May 1995, Ros 1995, Primack \& Ros 2002). En cuanto a la posible pérdida de hábitats, uno de los ecosistemas más amenazados en el país es el bosque mesófilo de montaña con su alta proporción de plantas y animales endémicos que abarca el 1\% de la superficie del país (Toledo \& Ordóñez 1993), de hecho en el Estado de Veracruz se ha perdido el $40 \%$ en las últimas dos décadas debido en gran parte a su conversión a potreros, cultivos (entre los que se encuentran los cafetales) y zonas urbanas (Williams-Linera et al. 2002).

Generalmente, la transformación de los hábitats naturales es provocada por un conjunto de factores sociales, económicos, y biológicos, que inducen la desaparición de especies vegetales y animales. Sin embargo, el cultivo del café de sombra por su sistema de manejo que implica la conservación de árboles, es menos negativo para la 
conservación de la biodiversidad, como lo comprueban los resultados obtenidos en el Proyecto BIOCAFE con el estudio de 12 grupos taxonómicos (Manson et al. 2008). El agroecosistema cafetalero permite que se mantengan diversas especies de mamíferos medianos (que tienen un peso que está en un rango de $500 \mathrm{~g} \mathrm{a} 10 \mathrm{~kg}$ ) y se encuentran alrededor de un $70 \%$ de las especies que originalmente existen en el bosque mesófilo de montaña, ver Gallina et al. (2008). Por el papel que desempeña cada especie en un ecosistema, se puede apreciar cual puede ser su impacto en la comunidad de estudio, y lo que puede afectar al ecosistema cuando desaparece alguna especie. Dentro de los posibles impactos de los mamíferos medianos sobre la comunidad vegetal y animal tenemos: la dispersión de semillas, la depredación de semillas y el control sobre las poblaciones de insectos y roedores (Gallina et al. 1992). De acuerdo al tipo de sistema de cultivo de café que se practique (1. El rústico, 2. policultivo tradicional, 3. policultivo comercial, 4. monocultivo bajo sombra, 5 . monocultivo sin sombra) se esperaría encontrar mayor diversidad de mamíferos en los cafetales que presenten características que permitan obtener los beneficios que encontrarían en su hábitat natural, dado que éste presentaría cobertura de escape, de traslado, de protección contra el clima, y de descanso durante el día, así como alimentación, que en este caso sería el rústico y el policultivo, en los cuales tenemos mayor diversidad vegetal mientras que en los tres últimos encontraríamos menos diversidad de organismos (Gallina et al. 2008). Actualmente el significado y la importancia de la biodiversidad no está en duda y se han desarrollado gran cantidad de parámetros para medirla como un indicador del estado de los sistemas ecológicos y para aplicarla con fines de conservación, manejo y monitoreo (Spellerberg 1991).

Sin embargo, aunque el agroecosistema cafetalero permite la conservación de una biodiversidad de mamíferos medianos, hoy en día la cacería es uno de los factores que está amenazando estos mamíferos. Generalmente los animales más frecuentemente cazados son aquellos comunes en el área y con tasas reproductivas altas como ha sido señalado por diversos autores (Bodmer 1995; Bennett \& Robinson 2000). Si bien en algunas áreas la cacería puede ser sustentable para algunas especies, no lo es para todas debido a que las poblaciones no responden de la misma manera a tal presión (Bodmer et al. 1997, Bennett \& Robinson 2000, Jorgenson 2000). Como señalan Robinson \& Redford (1994), la problemática que enfrentan las poblaciones de animales cazados es en muchos aspectos similar en las regiones tropicales de América Latina, junto con la deforestación y transformación de los bosque nativos, la cacería sin control parece estar afectando negativamente a numerosas especies, que de ser manejadas adecuadamente podrían tener un gran potencial de aprovechamiento (Robinson \& Redford 1994, Alvard et al.1997, Robinson \& Bodmer 1999, Robinson \& Bennett 2000).

La cacería es una actividad común de las poblaciones rurales cercanas a los cafetales que está mermando las poblaciones y la diversidad de mamíferos de la 
zona. Hoy en día existen 24 especies de mamíferos medianos dentro de los cafetales del centro de Veracruz (García 2007), de éstas, cuatro especies se encuentran en peligro de extinción según la NOM-059-SEMARNAT-2002. En consecuencia el objetivo del trabajo es generar información que se requiere acerca de qué animales son cazados, para detectar declives en la población que permita evaluar el impacto de la caza y establecer alternativas para un aprovechamiento sustentable de la fauna. Los objetivos particulares de este estudio fueron: 1) registrar las especies de mamíferos medianos cazadas en la zona centro de Veracruz, 2) estimar la cantidad de animales que se extraen, así como la biomasa que pueden estar aprovechando en la zona, y 3) caracterizar el tipo y forma de cacería; identificando los métodos de cacería, el uso que le dan a los organismos cazados, así como analizar si existe un patrón temporal de cacería, si tienen alguna preferencia en cuanto a estructura de edades y sexo, y la percepción que tienen los cazadores hacia las presas.

\section{Área de estudio}

\section{MATERIAL Y MÉTODOS}

Los sitios muestreados se encuentran entre Huatusco y Xalapa, Veracruz, entre los 1200 y 1340 msnm en el Eje Neovolcánico Transversal. La zona de estudio se encuentra comprendida dentro de las coordenadas UTM extremas: 708501 Norte, 2159117 Oeste y 724888 Norte, 2103753 Oeste. La precipitación anual varía entre 1350 y $2200 \mathrm{~mm}$, mientras que la temperatura media anual está entre los $12^{\circ}$ C y $\operatorname{los} 19.5^{\circ} \mathrm{C}$ (Manson et al. 1999). Hay tres estaciones bien definidas: la relativamente seca y fría de octubre-noviembre a marzo, una época seca cálida durante abril y mayo, y una lluviosa cálida de junio a septiembre (Williams-Linera 1992). La vegetación dominante dentro de los cafetales, comprende tanto especies nativas (Trema micrantha, Heliocarpus donnellsmithi, Alchornea latifolia, Trichilia havanensis, Enterolobium cyclocarpum, Rapanea myricoides y Bursera simaruba), como especies introducidas tal como bracatinga (Mimosa scabrella), cítricos, plátano y otras). Williams-Linera et al. (2005) mencionan como ejemplos de especies dominantes del bosque mesófilo de la región a las siguientes: Liquidambar sytraciflua, Platanus mexicanus, Carpinus caroliniana, Clethra mexicana, Quercus germana, Q. leiophylla, Q. xalapensis y Turpinia insignis. Entre los arbustos están Eugenia xalapensis, Hoffmania excelsa y Palicourea padifolia (Castillo-Campos 1991) (Fig.1).

La selección de las fincas cafetaleras a muestrear fueron seis y nos basamos en dos características: 1) cafetales estudiados dentro del proyecto BIOCAFE (Manson et al. 2008), y 2) evidencias de cacería practicada regularmente por los pobladores locales. 


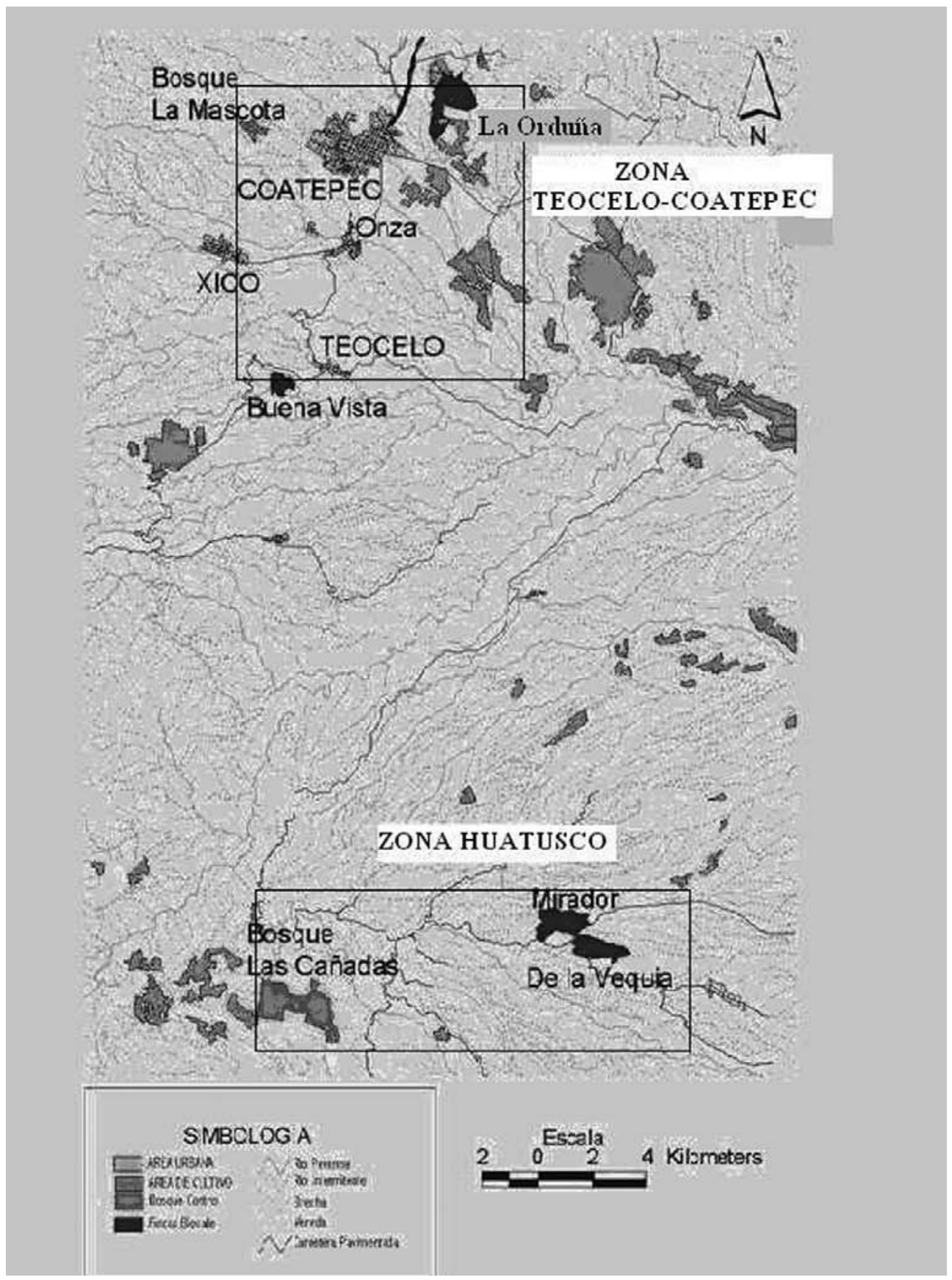

Figura 1. Localización geográfica del área de estudio. 
Para realizar el estudio dividimos los cafetales en dos zonas, esto debido a que los cazadores utilizan diferentes cafetales según la cercanía entre ellos. La primera zona fue Teocelo-Coatepec, en la cual nos enfocamos en cuatro fincas: 1) La Onza es una finca de tipo policultivo, con árboles no mayores a $10 \mathrm{~m}$. Se pueden encontrar cítricos, plátano, bracatinga, árboles frutales e Inga spp. 2) La Orduña es una finca de tipo policultivo. Se pueden encontrar árboles mayores a $15 \mathrm{~m}$, Ficus sp., plátanos, cítricos, Inga spp., nacaztle (Enterolobium cyclocarpum) y casuarinas. 3) Teocelo Sol, es la única finca con tratamiento bajo sol, por lo tanto no se encuentra ningún árbol sembrado para sombra. 4) La Mascota es un fragmento de bosque mesófilo de montaña, aunque se pueden encontrar árboles mayores a 15 m, Ficus sp., plátanos, cítricos, Inga spp., nacaztle y casuarinas.

La segunda zona fue Huatusco en la cual solo se trabajó en dos fincas: 1) El Mirador, que tiene un tipo de manejo rústico con cierto grado de policultivo tradicional, colinda con áreas de bosque relativamente conservado, con árboles mayores a los $10 \mathrm{~m}$ de altura. 2) Finca de La Vequia, esta finca está rodeada de cañaverales, el cultivo es de tipo sombra especializada cuya cubierta es mayormente Inga vera y Eritrina sp. La cubierta vegetal tiene una altura menor de $10 \mathrm{~m}$, con cafetos grandes de alrededor de $2 \mathrm{~m}$.

\section{Trabajo de campo}

Las entrevistas estructuradas se llevaron a cabo durante los meses de enero a mayo de 2006, aplicándose a personas que regularmente cazan y que se encontraban en poblados y ciudades aproximadamente dentro de un radio de 3 kilómetros alrededor de las fincas cafetaleras seleccionadas. El análisis de resultados de las entrevistas se realizó tomando en cuenta la frecuencia de respuestas dadas por los cazadores. Las entrevistas abarcaron 24 preguntas (Anexo), con lo que se obtuvo información referente a cuantos animales cazan por unidad de tiempo, si hay preferencias por sexos y edades de los animales, los métodos que usan, usos que le dan a los animales cazados, si causan algún daño a la comunidad y la biomasa extraída por cazador (kg obtenidos por especie cazada, estimada a partir del peso promedio de cada especie por el número de animales cazados de esa especie). Para realizar la estimación de la presión de cacería se tomó en cuenta el número de individuos cazados por año entre el tamaño del área de estudio:

$$
P c=\frac{I t}{A t}
$$

En donde: $\mathrm{Pc}=$ presión de cacería, $\mathrm{It}=$ total de individuos cazados al año y At $=$ área total en donde se realizó la cacería.

Se transformaron los datos de porcentaje de animales cazados (por especie) y se aplicó una prueba de t para muestras pareadas para saber si había diferencias 
significativas. En cuanto a la biomasa extraída estimada, se cálculo obteniendo el número total de individuos de las especies cazadas anualmente de acuerdo a los resultados de la entrevista, y este número fue multiplicado por el peso promedio de cada especie y se utilizó un ANOVA de una vía para conocer si existían diferencias en las dos áreas muestreadas.

\section{RESULTADOS}

\section{Mamíferos cazados en las zonas de estudio}

Realizamos un total de 77 entrevistas, registrando 16 especies cazadas para ambas zonas, siendo el armadillo, el conejo, el mapache y el tlacuache los de mayor demanda. Para la zona de Teocelo-Coatepec corresponde el mayor número de entrevistas realizadas (63) registrando 13 especies de mamíferos cazadas. Para la zona Huatusco efectuamos solo 14 entrevistas registrando 11 especies de mamíferos cazados, en donde al igual que en la zona Teocelo-Coatepec, el armadillo encabeza la lista (Cuadro 1). Los datos en porcentaje se transformaron a arcoseno y el resultado de la prueba de t-pareada mostró que no fueron significativos $(\mathrm{T}=-0.27$, g.l. $=12, \mathrm{P}=0.79)$.

Cuadro 1. Número de individuos cazados por especie de acuerdo a las entrevistas y el porcentaje que representan en las dos áreas de estudio en el centro de Veracruz.

\begin{tabular}{llrrrr}
\hline \multicolumn{1}{c}{ ESPECIE } & \multicolumn{2}{c}{ HUATUSCO } & \multicolumn{2}{c}{ TEOCELO-COATEPEC } \\
\hline & & No. de & $\%$ & $\begin{array}{c}\text { No. de } \\
\text { individuos }\end{array}$ & $\%$ \\
\hline Ardilla & Sciurus aureogaster o S. deppei & 0 & 0 & 462 & 8.78 \\
Armadillo & Dasypus novemcinctus & 214 & 32.67 & 1806 & 34.32 \\
Cacomixtle & Bassariscus astutus o B. sumichrasti & 0 & 0 & 141 & 2.68 \\
Chupamiel & Tamandua mexicana & 0 & 0 & 1 & 0.02 \\
Comadreja & Mustela frenata & 0 & 0 & 30 & 0.57 \\
Conejo & Sylvilagus floridanus & 40 & 6.11 & 1659 & 31.53 \\
Coyote & Canis latrans & 0 & 0 & 4 & 0.08 \\
Cuaqueche & Dasyprocta mexicana & 28 & 4.27 & 0 & 0 \\
Mapache & Procyon lotor & 45 & 6.87 & 365 & 6.94 \\
Martucha & Potos flavus & 1 & 0.15 & 0 & 0 \\
Tejon & Nasua narica & 29 & 4.43 & 0 & 0 \\
Tigrillo & Leopardus wiedii & 5 & 0.76 & 3 & 0.06 \\
Tlacuache & Didelphis virginiana & 190 & 29.01 & 585 & 11.12 \\
Tuza real & Cuniculus paca & 3 & 0.46 & 0 & 0 \\
Zorra & Urocyon cinereoargenteus & 91 & 13.89 & 204 & 3.88 \\
Zorrillo & Mephitis macroura & 9 & 1.37 & 2 & 0.04 \\
TOTAL & & 655 & 100 & 5262 & 100 \\
\hline & & & &
\end{tabular}




\section{Métodos de caza}

Los cazadores de la zona Teocelo-Coatepec emplearon principalmente cuatro métodos para cazar: perro (41\%) en este método los cazadores sueltan de cuatro a siete perros y esperan a que éstos atrapen a la presa; trampa rústica $(18 \%)$ que colocan en veredas y cerca de cuerpos de agua y consiste en poner sobre cuatro palos una piedra de gran tamaño la cual cae sobre la presa, matándola de un golpe; trampas de alambre (18\%) las cuales son trampas parecidas a las trampas Tomahawk; y arma de fuego (16\%) este método puede ser utilizado en el día o en la noche, y los cazadores esperan a su presa cerca de cuerpos de agua y cuando los tienen cerca, les disparan. En cuanto a la zona de Huatusco obtuvimos que dos son los métodos más usados: el arma de fuego que representa el $53 \%$ y la arreada con un 33\%; este método es nocturno y consiste en acorralar a la presa con los perros, utilizando lámparas y armas de fuego, seguidos de la trampa rústica con un $18 \%$ (Fig. 2).

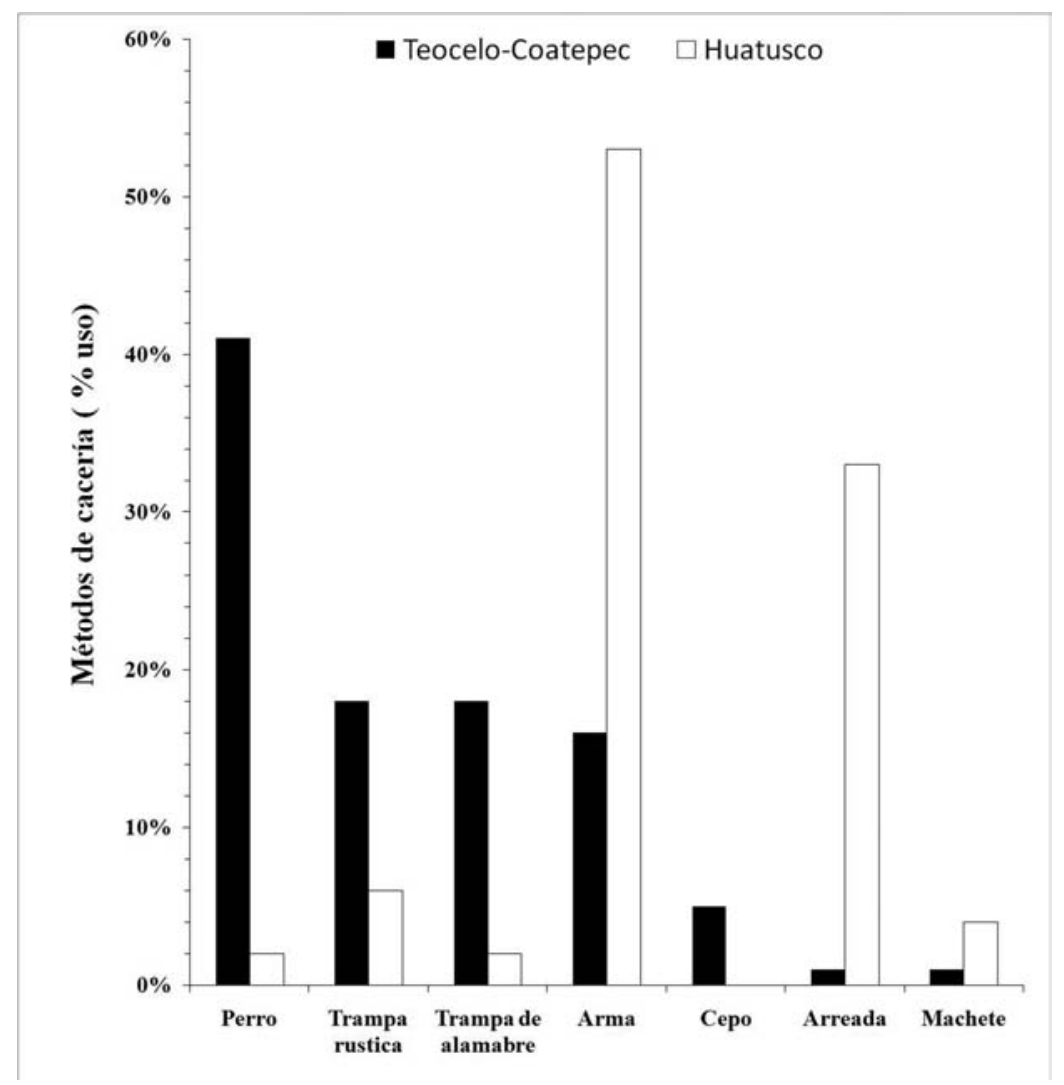

Figura 2. Métodos de cacería más comunes en las zonas Huatusco y Teocelo-Coatepec. 


\section{Preferencia en cuanto a estructura de edades de la presa}

A pesar de que en ambas zonas los cazadores mencionan que prefieren las presas adultas, sobre las crías o juveniles, en la zona Teocelo-Coatepec, los cazadores señalan que es difícil realizar esta selección debido a que al utilizar los perros, éstos atrapan al primer animal que ven y muchas de las veces éstos son crías. En la zona de Huatusco generalmente cazan con arma de fuego y esto les permite muchas veces elegir una presa de mayor tamaño.

\section{Uso de animales cazados}

Para la zona Teocelo-Coatepec obtuvimos que de las 13 especies cazadas sólo siete son utilizadas como alimento: ardilla, armadillo, conejo, mapache, chupamiel (Tamandua mexicana), tejón y tlacuache. Las especies de uso comercial son ocho (ardilla, armadillo, cacomixtle, tejón, conejo, coyote (Canis latrans), mapache y tlacuache). Sin embargo, hay que recalcar que a pesar de ser más especies con este uso, son pocos los cazadores que se dedican a cazar con fines comerciales, y regularmente en el caso del armadillo y el conejo son vendidos a los habitantes de la misma localidad para consumo humano. En cuanto al uso medicinal sólo tenemos tres especies: el coyote, el armadillo y el zorrillo. La mayoría de las especies utilizadas como alimento tienen también uso ornamental, y tenemos registradas diez especies con este uso. Un importante dato es el que obtuvimos sobre la zorra, puesto que ésta es cazada para evitar daños a sus aves de corral, para entrenar perros de cacería y como diversión.

En la zona Huatusco se observa una distribución más homogénea en cuanto al uso alimentario puesto que de las once especies registradas, diez son utilizadas para alimento, solo la zorra es cazada para evitar daños, y para entrenar perros de cacería. En cuanto a animales que usan como medicinales, son los mismos en la zona Teocelo-Coatepec. Se observa una diferencia en cuanto a las especies que son cazadas para evitar daños ya que son cuatro las que figuran aquí: el mapache, tejón, zorra y tlacuache.

\section{Percepción de los cazadores hacia sus presas}

En la zona Teocelo-Coatepec, los cazadores mencionaban en general que la mayoría de los animales son muy abundantes en la zona a excepción del coyote, el chupamiel y el tigrillo a los cuales calificaron de escasos. En la zona Huatusco estos dos últimos dijeron no haberlos visto en el área en los últimos 4 años. Para el caso del zorrillo en la zona Teocelo-Coatepec es menos abundante según los cazadores que en la zona de Huatusco. En la zona Huatusco ningún animal fue calificado de muy abundante, el tlacuache, la martucha y la zorra, fueron las únicas especies que calificaron como abundantes (Fig. 3). 


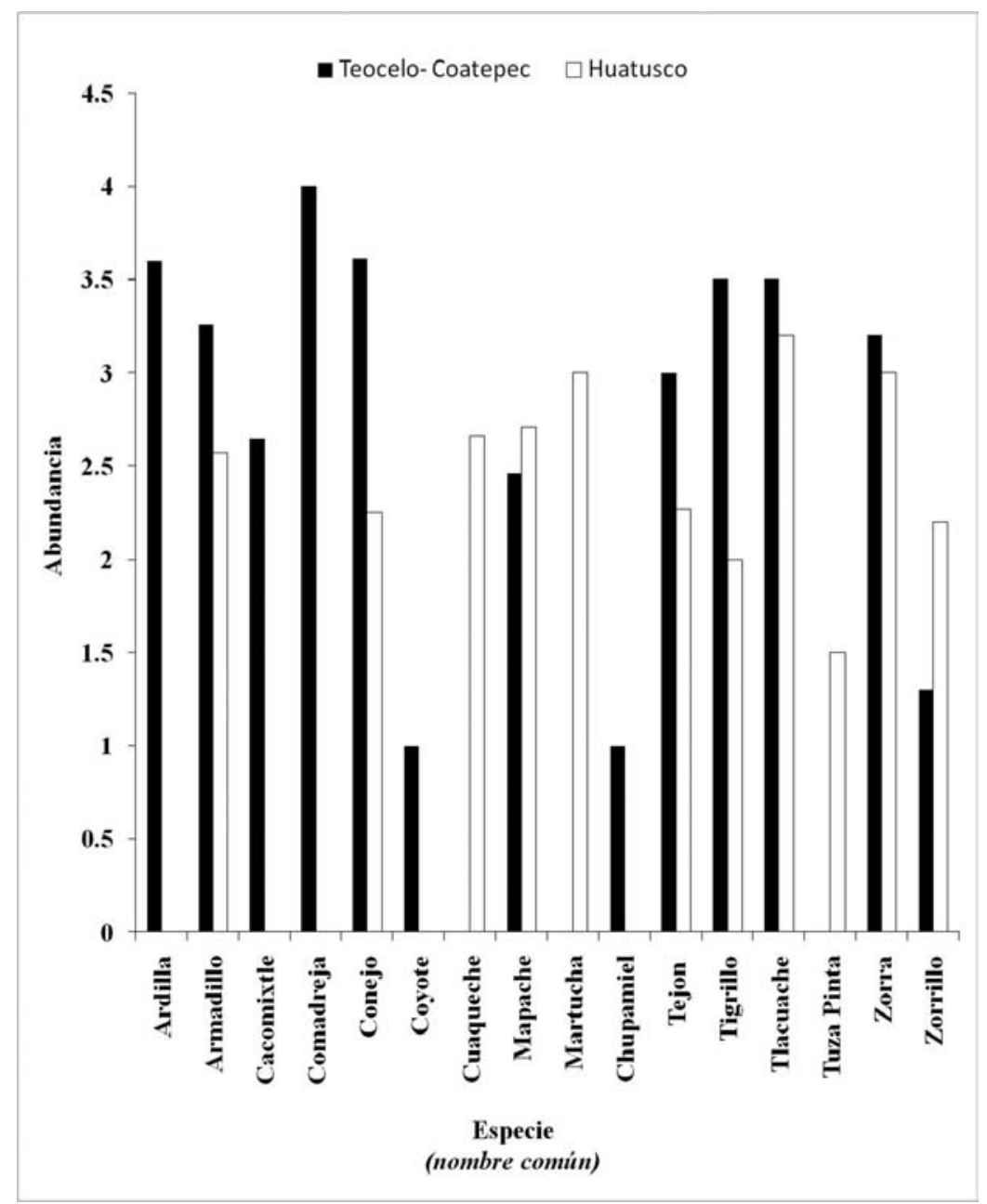

Figura 3. Abundancia de presas según los cazadores entrevistados: 4=Muy Abundante, $3=$ Abundante, $2=$ Regular, 1=Escaso. El valor cero significa que no lo han visto en los últimos cuatro años, a excepción de la ardilla la cuál es muy abundante pero no la cazan.

En el Cuadro 2 podemos apreciar que las especies más cazadas concuerdan con las más abundantes de acuerdo al estudio que realizaron Gallina et al. (1996).

\section{Biomasa extraída al año}

Los resultados como se muestran en la Cuadro 3 indican claramente mayor consumo del armadillo en la zona Teocelo-Coatepec pues mientras aquí se extrae un estimado 
Cuadro 2. Listado taxonómico de los mamíferos medianos de la región (tomado de Gallina et al. 1996; 2008) SIMBOLOGIA: Locomoción: T=Terrestre, E= Escansorial, A= Arborícola, $\mathrm{Ac}=$ Acuático; Forrajeo: F=Frugívoro, $\mathrm{O}=$ Omnívoro, $\mathrm{I}=$ Insectívoro, $\mathrm{M}=$ Mimercófago, $\mathrm{H}=$ Herbívoro, $\mathrm{G}=$ Granívoro y $\mathrm{C}=$ Carnívoro; Abundancia: $\mathrm{A}=$ Abundante, $\mathrm{C}=$ Común y $\mathrm{R}=\mathrm{Rara}$; Categoría de riesgo: $\mathrm{P}=$ Peligro de extinción, $\mathrm{Pr}=$ Sujeta a protección especial, $\mathrm{A}=\mathrm{Amenazada}$.

\begin{tabular}{|c|c|c|c|c|}
\hline Especie & Locomoción & Abundancia & Estatus & Peso (g) \\
\hline \multicolumn{5}{|l|}{ MARSUPIALES } \\
\hline Didelphis marsupialis & $\mathrm{E}$ & $\mathrm{A}$ & & 1041 \\
\hline Didelphis virginiana & $\mathrm{E}$ & A & & 2300 \\
\hline Philander opossum & $\mathrm{T}$ & A & & 400 \\
\hline \multicolumn{5}{|l|}{ EDENTADOS } \\
\hline Dasypus novemcinctus & $\mathrm{T}$ & A & & 3544 \\
\hline Tamandua mexicana & A & $\mathrm{R}$ & $\mathrm{P}$ & 4210 \\
\hline \multicolumn{5}{|l|}{ ROEDORES } \\
\hline Sciurus aureogaster & A & A & & 338 \\
\hline Sciurus deppei & A & $\mathrm{A}$ & & 220 \\
\hline Orthogeomys mexicana & $\mathrm{F}$ & $\mathrm{C}$ & & 100 \\
\hline Sphiggurus mexicanus & A & $\mathrm{R}$ & $\mathrm{A}$ & 1000 \\
\hline Dasyprocta mexicana & $\mathrm{A}$ & $\mathrm{R}$ & & 3600 \\
\hline Agouti o Cuniculus paca & A & $\mathrm{R}$ & & 8227 \\
\hline \multicolumn{5}{|l|}{ LAGOMORFOS } \\
\hline Sylvilagus floridanus & $\mathrm{T}$ & $\mathrm{C}$ & & 1025 \\
\hline \multicolumn{5}{|l|}{ CARNIVOROS } \\
\hline Canis latrans & $\mathrm{T}$ & $\mathrm{R}$ & & 10000 \\
\hline Urocyon cinereoargenteus & $\mathrm{T}$ & $\mathrm{C}$ & & 5500 \\
\hline Puma yaguaroundi & $\mathrm{T}$ & $\mathrm{R}$ & A & 5000 \\
\hline Leopardus wiedii & A & $\mathrm{R}$ & $\mathrm{P}$ & 3500 \\
\hline Mephitis macroura & $\mathrm{T}$ & $\mathrm{R}$ & & 2500 \\
\hline Galictis vittata & $\mathrm{T}$ & $\mathrm{R}$ & & 2910 \\
\hline Mustela frenata & $\mathrm{T}$ & $\mathrm{C}$ & & 450 \\
\hline Potos flavus & $\mathrm{A}$ & $\mathrm{R}$ & $\operatorname{Pr}$ & 2490 \\
\hline Bassariscus astutus & $\mathrm{E}$ & $\mathrm{C}$ & & 1200 \\
\hline Bassariscus sumichrasti & $\mathrm{E}$ & $\mathrm{C}$ & & 1400 \\
\hline Nasua narica & $\mathrm{E}$ & A & & 3380 \\
\hline Procyon lotor & $\mathrm{E}$ & $\mathrm{C}$ & & 8850 \\
\hline
\end{tabular}

de $125.6 \mathrm{~kg} / \mathrm{cazador}$ al año, en la zona Huatusco fueron $57.35 \mathrm{~kg} / \mathrm{cazador}$, existiendo un diferencia de $68.3 \mathrm{~kg}$. En la zona Huatusco, se observan datos más homogéneos en cuanto a los kilos consumidos y la diversidad de especies. Por su parte la zona Teocelo-Coatepec el mayor consumo recae sobre el armadillo y las cuatro especies restantes son consumidas en proporciones mucho menores. Sin embargo, al aplicar el ANOVA de una vía no se encontraron diferencias significativas al comparar las dos zonas $(\mathrm{F}=0.22$, g.1. $=1,18, \mathrm{P}=0.88)$. 
Tlapaya \& Gallina: Cacería de mamíferos en cafetales

Cuadro 3. Se representa la biomasa estraida al año de acuerdo al número de individuos que se cazan al año y por cazador en la zona Teoceolo-Coatepec y Huatusco, de acuerdo con la estrevista.

\begin{tabular}{lrrrr}
\hline & \multicolumn{2}{c}{ Zona } & \multicolumn{2}{c}{ Zona } \\
& Teocelo-Coatepec & \multicolumn{2}{c}{ Huatusco } \\
\hline Especie & kg totales & kg./cazador & kg totales & kg./cazador \\
\hline Dasypus novemcinctus & 7917 & 125.6 & 803 & 57.3 \\
Didelphis spp. & 1613 & 25.6 & 576 & 41.1 \\
& & & & \\
Sylvilagus floridanus & 1438 & 22.8 & 300 & 21.4 \\
Procyon lotor & 1073 & 17 & 312 & 22.2 \\
Nasua narica & 108 & 1.7 & 204 & 14.5 \\
& & & & \\
Sciurus spp. & 60 & 0.95 & 0 & 0 \\
Tamandua mexicana & 15 & 0.23 & 0 & 13.1 \\
Dasyprocta mexicana & 0 & 0 & 184 & 2.42 \\
Cuniculus paca & 0 & 0 & 34 & 1.71 \\
Mephitis macroura & 0 & 0 & 24 & 174 \\
Total & 12224 & 194 & 2437 & \\
\hline
\end{tabular}

\section{Presión de cacería}

Para evaluar la presión se tomó en cuenta el número de individuos cazados por año entre el tamaño del área de estudio (Cuadro 4), siendo mayor para la zona de Teocelo-Coatepec con un valor de 14.68 y para la zona de Huatusco de 2.54.

Cuadro 4. Estimación de la presión de cacería en las dos zonas de estudio: ZonaTeocelo-Coatepec y Zona Huatusco.

\begin{tabular}{lcccc}
\hline & Individuos/Cazador & Individuos/año & Área(ha) & Individuos/ ha/año \\
\hline Teocelo -Coatepec & 88 & 5554 & 378.24 & 14.684 \\
Huatusco & 46 & 642 & 252.48 & 2.543 \\
\hline
\end{tabular}

\section{DISCUSIÓN}

En la región central de Veracruz, existe estudios que revelan el empobrecimiento de la fauna, González-Romero \& López-González (1993) encontraron que en los 
últimos 27 años, la diversidad de mamíferos en la región de Xalapa y sus alrededores, se redujo en un $54 \%$ debido a la urbanización y a las actividades agropecuarias. Contrariamente a esto, se carece de información sobre la cacería furtiva y como está influye en este proceso. Los métodos que comúnmente se usan para obtener datos sobre esta actividad son las entrevistas estructuradas.

La selección de la entrevista estructurada como un método de investigación tiene ventajas y desventajas siendo especialmente sensible y efectiva para dar respuestas a cierto tipo de preguntas. En este trabajo en particular se obtuvo una presión de caza aproximada, en cambio se encontraron datos muy útiles, como lo son el registro de especies presentes en el área de estudio y los métodos de caza utilizados. Las especies encontradas concuerdan con otros estudios realizados en la zona por García (2007) y Gallina et al. (1996, 2008). De las 16 especies registradas por medio de la encuesta, nueve son cazadas en cantidades importantes y siete representaron menos del $1 \%$ en ambas zonas. Bennett et al. (2000) señalan que los animales más frecuentemente cazados son aquellos comunes en el área y con tasas reproductivas altas. Así, en el estudio realizado por Gallina et al. (1996) las especies que se encontraron como más abundantes, concuerdan con nuestros resultados sobre las especies más cazadas que son el armadillo, conejo, tlacuache, ardilla, mapache, zorra, cacomixtle, tejón y cuaqueche. Estos resultados fueron similares al compararlos con los obtenidos por Lira (2005) en la Tuza Monroy, Oaxaca, en donde el armadillo y el tejón resultan ser de las especies más apreciadas por los cazadores de esa zona, en tanto que Guerra \& Naranjo (2003) en la zona de la selva Lacandona encuentran también que el armadillo fue de las especies con mayor tasa de extracción (0.43 individuos/kilómetro/año)

En cuanto al método utilizado para cazar se encontraron dos que son los más aplicados. En la zona Teocelo-Coatepec sólo el 16\% utilizan arma de fuego, predominando el uso de perros $(41 \%)$, lo que no permite hacer una selección de individuos en cuanto a edad y tamaño. Por otra parte en zona Huatusco el 53\% de los cazadores cazan con arma de fuego, lo cual sí les permite seleccionar individuos de tamaño grande lo que implica generalmente que sean animales adultos. Estos métodos para cazar son similares a los reportados por Guerra \& Naranjo (2003) en la selva Lacandona, encontrando que el 56\% de los cazadores usan arma de fuego y el $22 \%$ usa perros entrenados, por su parte Lira (2005) observó tres tipos de cacería; la arreada, la caza en cayuco, y la espera en áreas de alimentación en donde solo se utiliza arma de fuego. Sin embargo los resultados en cuanto a la relación de los métodos utilizados con el número de individuos cazados difiere con lo reportado por Alvard et al. (1996) quienes mencionan que el uso del arma de fuego en la cacería permite obtener un mayor número de individuos por cazador, a diferencia de los cazadores con arco. Por nuestra parte, se comparó el uso de arma de fuego con el perro entrenado para cazar y encontramos que para la zona Teocelo-Coatepec, en 
donde se caza con perro, se capturan aproximadamente 88 individuos al año/cazador, mientras que para la zona de Huatusco en donde se caza con arma de fuego se capturan alrededor de 46 individuos al año/cazador. Los cazadores de la zona Teocelo-Coatepec nos comentaron que con perro pueden cazar hasta 5 individuos por noche, pero esto incluye a cualquier animal comestible o no. Es por eso que existen muchos animales que son cazados por los perros, sin embargo éstos pueden no ser aprovechados por los cazadores, por ejemplo, el tlacuache "apestoso" (Didelphis marsupialis) que no lo come la gente por su sabor fuerte y desagradable a diferencia de la otra especie $D$. virginiana que sí se utiliza como alimento y cuyas poblaciones son menos abundantes que la primera mencionada, pudiendo ser un efecto directo de la cacería (Gallina et al. 2008). Por ejemplo, en un estudio realizado sobre hábitos alimentarios y reproducción de las dos especies de tlacuaches de junio de 2003 a mayo de 2005 en los mismos cafetales, Oceguera-González \& González-Romero (2008) capturaron 25 D. virginiana y 45 D. marsupialis.

La preferencia con respecto a las edades de los individuos cazados está relacionada con el método de cacería. Cuando la cacería es realizada con arma de fuego, es posible seleccionar el tamaño de la presa, cuando la cacería se realiza con perros no existe esta selección, incluso existe el problema que los perros pueden dejar malheridos a los animales que acaban luego muriendo sin ser aprovechados. También influye el fin con el que fue cazado el organismo. Si se considera a la presa como una plaga, que es el caso de la zorra, y el tlacuache (Didelphis marsupialis), no existe discriminación de la edad. La selección de sexos, macho o hembra, generalmente no existe debido a que en la mayoría de los animales cazados no tienen dimorfismo sexual marcado con el cual los cazadores puedan guiarse. Sin embargo en el caso del tejón, donde sí se llegan a distinguir el macho de la hembra, prefieren al macho debido a que es de mayor tamaño y por lo tanto tiene más carne. Los entrevistados se refieren al tejón como tejón de manada (incluye las hembras con juveniles) y al tejón solo (machos adultos y viejos).

Respecto a los usos que le dan a las especies, en la zona de Teocelo-Coatepec hay una mayor diversidad de usos. Los que tiene mayor aprovechamiento son el armadillo, la ardilla, el mapache y el conejo, que son usados como alimento, medicina, ornamental, comercial, artesanal, entre otros. Para la zona de Huatusco tenemos un panorama menos diverso en cuanto a usos. Nueve de las once especies cazadas son aprovechadas exclusivamente para alimento.

También se pudo registrar que en ambas zonas existen especies que son cazadas pero no son aprovechadas como es el caso de la zorra y la comadreja, a las que se les considera dañinas. También está el caso del yaguarundi que sólo es cazado por diversión. En el caso particular de la zorra la cual tiene mayor demanda en la zona de Huatusco, podemos decir que aunque es considerada plaga y dañina, para ambas zonas la información muestra que ésta es una especie de gran importancia como lo 
señalan Primack \& Ros (2002) quienes mencionan que los depredadores situados en la cúspide de las cadenas tróficas como los zorros, son las especies clave más evidentes porque a menudo son importantes en el control de las poblaciones de herbívoros como roedores y lagomorfos. Sin zorros, las poblaciones de herbívoros suelen aumentar, provocando un sobrepastoreo, pérdida de biomasa vegetal, la pérdida consiguiente de insectos y la erosión del suelo, e inclusive pueden volverse plaga en algunos cultivos.

En su mayoría los cazadores son locales y consideran que sí ha habido una disminución de los mamíferos, en especial del chupamiel, mapache y tejón, debido a la cacería excesiva y, a que algunos cazadores no respetan vedas (época de apareamiento y reproducción). También señalan que como se van asentando más viviendas, los animales se van alejando hacia zonas menos pobladas, ya que muchas veces son atacados por perros y por los pobladores. Los cazadores locales admiten que los mamíferos son importantes para la comunidad debido a que es una fuente de alimento económico. También señalan la importancia de cazar sólo organismos adultos. En el caso de los cazadores que matan por diversión, en su mayoría dicen que la cacería es un método de control.

La disminución de las poblaciones de la fauna silvestre está relacionada con la destrucción de su hábitat por deforestación y la caza excesiva (Dirzo \& Miranda 1990, 1991). En el caso específico de la zona centro de Veracruz, la caza y las actividades humanas podrían estar repercutiendo en las poblaciones de animales que habitan, pues los entrevistados mencionaron que los mamíferos de tamaño mayor como la tuza real y el chupamiel se han retirado hacia zonas menos pobladas y con mayor vegetación nativa. Además de la reducción de la fauna, la cacería puede desembocar en otros efectos sobre la flora y fauna; como lo señalan Stoner et al. (2007) quienes evaluaron el efecto indirecto de la cacería para bosques tropicales, y mencionan el efecto negativo sobre la comunidad de plantas y de invertebrados.

La estimación de biomasa extraída al año fue mayor para la zona de TeoceloCoatepec con un valor de $194 \mathrm{~kg} /$ cazador de los cuales $125 \mathrm{~kg}$ corresponde al armadillo. En la zona Huatusco se obtuvo un valor de $174 \mathrm{~kg} / \mathrm{cazador}$. En esta zona se observan datos más homogéneos en cuanto a las cantidades de las diversas presas consumidas. En relación a la estimación de la presión de caza, ésta fue mucho mayor para la zona de Teocelo-Coatepec en donde obtuvimos un valor de 14.68 individuos /ha/año, mientras que para la zona de Huatusco se estimó un valor de 2.54 individuos /ha/año, debido a la menor población humana que lleva a cabo esta actividad. La información disponible indica que cuando las poblaciones humanas son pequeñas, la presión de la caza no es severa. Grandes porciones donde se encuentran los cafetales, junto con parches de bosque de niebla menos alterados bastan para amortiguar y reabastecer la región que tiene actividad concentrada. Esto es un claro ejemplo de lo 
que ocurre en la zona Huatusco pues existen pequeños grupos dispersos de 15 a 20 casas, con necesidades de caza moderadas, que permiten que se mantengan las poblaciones de mamíferos medianos.

La actividad de caza que se practica en ambas zonas es completamente ilegal, ninguno de los cazador entrevistados contaba con un permiso de cacería, es por esto que es necesario realizar planes de manejo de fauna. La figura jurídica bajo la cual se da el manejo de la fauna en México son las Unidades para la Conservación, Manejo y Aprovechamiento de la Vida Silvestre (UMA), las cuales son de dos tipos: extensivas e intensivas. Las primeras consisten en el manejo de poblaciones silvestres. Para este tipo de UMA es necesario calcular si la cacería es sostenible bajo las condiciones actuales. Para esto, es necesario llevar a cabo estudios de las poblaciones de los mamíferos y poder establecer cuotas de captura. Otra alternativa es reproducir los animales de mayor demanda en cautiverio, mediante UMAs intensivas. Esto podría representar una alternativa económica para las comunidades. Estas acciones promoverían el manejo y la conservación de las especies y al mismo tiempo contribuiría al desarrollo de la comunidad en el área de estudio.

Nuestros resultados mostraron que de las 16 especies cazadas, el armadillo es el animal que más se caza y el más aprovechado en ambas zonas. El armadillo fue la especie con mayor demanda, ya que además de ser un alimento preferido por las comunidades, también es considerado un remedio eficaz para las enfermedades respiratorias. En la zona de Teocelo-Coatepec tenemos un panorama de usos más diversos mientras que para la zona de Huatusco se aprecia que la mayoría de las presas son cazadas con fines alimenticios. La presión de cacería fue mayor para la zona de Teocelo-Coatepec, esto por diversos factores como lo son el método de cacería utilizado, y aunado a esto que esta zona es la más habitada y es más fácil acceder a los cafetales, por lo que la biodiversidad de mamíferos medianos está seriamente amenazada y podemos señalar que su conservación está en riesgo. De los datos anteriores surgen dos interrogantes que necesitan estudios a largo plazo: ¿Cuánto tiempo pueden soportar las poblaciones de mamíferos este ritmo de cacería? ¿Esta demanda está siendo sustentada por las poblaciones de mamíferos medianos en condiciones naturales?

AGRADECIMIENTOS. Este trabajo fue posible gracias al proyecto BIOCAFE apoyado por SEMARNAT a Proyecto 2002-C01-0194, así como a todos los dueños, encargados de las fincas y habitantes de las comunidades que nos apoyaron a contactar a los cazadores. Agradecemos particularmente a Jorge Müller quien nos abrió las puertas de su casa. A Jorge García Burgos compañero de campo por su apoyo incondicional. Mi sincero reconocimiento al Dr. Antonio Fernández Crispín de la Benemérita Universidad Autónoma de Puebla, por colaborar a mejorar sustancialmente la encuesta, así como al Dr. Jesús Martínez Vázquez. 


\section{LITERATURA CITADA}

Alvard, M.S., J.G. Robinson, K.H. Redford \& H. Kaplan. 1997. The sustainability of subsistence hunting in the neotropics. Conservation Biology, 11:977-982.

Bodmer, R. E. 1995. Managing Amazonian Wildlife: biological correlates of game choice by detribalized hunters. Ecological Applications, 5:87-877.

Castillo-Campos, G. 1991. Vegetación y flora del municipio de Xalapa. Instituto de Ecología. A. C. Xalapa, Veracruz, México.

Dirzo, R. 1990. La biodiversidad como crisis ecológica actual ¿qué sabemos? Ciencias, Número Especial 4:48-55.

Dirzo, R. \& A. Miranda. 1991. Altered pattern of herbivory and diversity in the forest understory defaunation. Pp. 273-287. In: P.P. Prince, T.M. Lewinshon, G.W. Fernandez \& W.W. Benson (Eds). Plant-animal interactions: evolutionary ecology in tropical and temperate regions. Wiley, N.Y.

Gallina, S., A. González-Romero \& R. Manson. 2008. Mamíferos pequeños y medianos. Pp. 161-180. In: R. Manson, V. Hernández-Ortíz, S. Gallina \& K. Mehltreter (Eds). Agroecosistemas cafetaleros de Veracruz: biodiversidad, manejo y conservación. INECOL, INE-SEMARNAT.

Gallina, S., S. Mandujano \& A. González-Romero. 1992. Importancia de los cafetales mixtos para la conservacion de la biodiversidad. Boletin Sociedad Veracruzana de Zoología, 2(2):11-17.

Gallina, S., S. Mandujano \& A. González-Romero. 1996. Conservation of mammalian biodiversity in coffee plantation of central Veracruz, Mexico. Agroforesty Systems, 33:13-17.

Garcia, B. 2007. Comparación de la riqueza de mamíferos medianos en un gradiente de manejo de cafetales del centro de Veracruz. Tesis de Maestría. Instituto de Ecologia, A.C. Xalapa, Veracruz, México.

Guerra, M. M. \& E. J. Naranjo. 2003. Cacería de subsistencia en dos localidades de la selva Lacandona, Chiapas, México. Pp. 339-344. In: O.R. Polanco (Eds). Manejo de fauna silvestre en Amazonia y Latinoamérica. Selección de trabajos V Congreso Internacional. CITES, Fundación Natura. Bogotá, Colombia.

González-Romero, A. \& C. A. López-González. 1993. Reconocimiento preliminar de la mastofauna asociada a las zonas suburbanas de Xalapa y Coatepec. Pp. 223-243. In: I. R. López-Moreno (Eds). Ecología urbana aplicada a la ciudad de Xalapa. Instituto de Ecología, A. C. y H. Ayuntamiento de Xalapa, Veracruz.

Jorgenson, J. P. 2000. Wildlife conservation and game harvest by maya hunters in Quintana Roo, México. Pp. 251-266. In: J.G. Robinson \& E.L. Bennett (Eds). Hunting for sustainability in Tropical Forest. Columbia University Press, N. Y.

Lira, T. I. 2005. Utilización de la fauna silvestre a nivel subsistencia en México. Memorias XXII Simposio Sobre Fauna Silvestre. Facultad de Medicina Veterinaria y Zootecnia-UNAM 6-15.

Manson R., V. Hernández-Ortíz, S. Gallina \& K. Mehltreter (Eds). 2008. Agroecosistemas cafetaleros de Veracruz: biodiversidad, manejo y conservación. INECOL, INE-SEMARNAT.

Manson, R. H., R. S. Ostfeld \& C. D. Canham. 1999. Responses of a small mammal community to heterogeneity along forest-old-field edges. Landscape Ecology, 14: 335-367.

Oceguera-González, A. \& A. González-Romero. 2008. Hábitos alimentarios y reproducción de dos especies de tlacuaches en cafetales del centro de Veracruz, México. Pp. 295-309. In: C. Lorenzo, E. Espinoza \& J. Ortega (Eds). Avances en el estudio de los mamíferos de México. Publicaciones Especiales. Vol. II. Asociación Mexicana de Mastozoología, A.C. México, D.F.

Primack, R.B. \& J. Ros. 2002. Introducción a la biología de la conservación. Ariel Ciencia, Barcelona.

Reid, W.V. \& K.R. Miller. 1989. Keeping options the scientific basic for conserving biodiversity. World Resources Institute, Organization of American States, Washington. 
Robinson, J.G. \& K.H. Redford. 1994. Measuring the sustainability of hunting in tropical forest. Oryx, 28:249-256.

Spellerberg, I.F. 1991. Monitoring ecological change. Cambridge University Press, UK.

Stoner, E.K., K. Vulinec, J.S. Wright \& C.A. Peres. 2007. Hunting and plant community dynamics in tropical forests: A synthesis and future directions. Biotropica, 39(3): 385-397.

Toledo, V. M. \& M. de J. Ordoñez. 1993. The biodiversity scenario of Mexico: a review of terrestrial habitats. Pp. 757-777. In: T.P. Ramamoorthy, R. Bye, A. Lot \& J. Fa (Eds). Biological diversity of Mexico. Origins and distribution. Oxford University Press. N.Y.

Williams-Linera, G. 2002. Tree species richness complementarity, disturbance and fragmentation in a Mexican tropical montane cloud forest. Biology and Conservation, 11:1825-1843.

Williams-Linera, G., A.M. López-Gómez \& M.A. Muñiz-Castro. 2005. Complementariedad y patrones de anidamiento de especies de árboles en el paisaje de bosque mesófilo del centro de Veracruz, México. Pp. 153-164. In: G. Halffter, J. Soberon, P. Koleff \& A. Melic (Eds). Sobre diversidad biológica: el significado de la diversidades Alfa, Beta y Gamma, Monografías Tercer Milenio, Vol. 4. SEA, CONABIO, Grupo DIVERSITAS \& CONACyT, Zaragoza. España.

Williams-Linera, G., R.H. Manson \& E. Isunza Vera. 2002. La fragmentación del bosque mesófilo de montaña y patrones de uso del suelo en la región oeste de Xalapa, Veracruz, México. Maderas y Bosques, 8(1):73-89.

http://www.conabio.gob.mx/informacion/catalogo_autoridades/NOM-059-SEMARNAT-2001/NOM059-SEMARNAT-2001.pdf 
Apéndice. Encuesta realizada a cazadores.

\section{ENTREVISTA SOBRE CACERÍA DE MAMÍFEROS EN CAFETALES}

Fecha 1. Ubicación

2. Distancia de la localidad al cafetal 3. Evidencias de cacería

DATOS DEL CAZADOR
4. Edad 5. Sexo 6. Ocupación
7. Especies cazadas

DATOS DE LAS PRESAS

Especie

1. Sexo MACHO HEMBRA

2. Edad
A) Crías
B) juveniles
C) Adultos
D) No sabe

3. Usos A) Alimenticio B) Comercial C) Medicinal D) Ornamental E) Artesanal F) Para evitar daños G) Diversión H) El perro lo mató

4. Métodos

5. Instrumentos que usa para la caza

6. Sitio donde caza A) Dentro del cafetal

7. Temporada de Cacería

B) Alrededores

9. Frecuencia de las salidas de cacería

10. ¿Cuántos animales caza por salida?

11. ¿Cómo considera a la especie que caza?
A) Muy abundante
B) Abundante
C) Regular
D) Escasa

12. ¿En qué zonas los ha visto? A) Dentro

B) Fuera del cafetal

13. ¿Cuántos estima que caza por año?

14. ¿Ha tenido usted problemas con los mamíferos?

15. Cada cuando come carne de monte A) 2 veces a la semana B) 1 vez a la semana C) 1 vez al mes

D) 1 vez cada 3 meses E) 1 vez al año

16. Cuántos kilos aproximadamente de carne de monte consume

Especie

1. Sexo MACHO HEMBRA

2. Edad
A) Crías
B) juveniles
C) Adultos
D) No sabe

3. Usos A) Alimenticio B) Comercial C) Medicinal D) Ornamental E) Artesanal
F) Para evitar daños G) Diversión
H) El perro lo mato

4. Métodos

5. Instrumentos que usa para la caza

6. Sitio donde caza A) Dentro del cafetal

7. Temporada de Cacería

B) Alrededores

9. Frecuencia de las salidas de cacería

10. ¿Cuántos animales caza por salida?

11. ¿Cómo considera a la especie que caza?
A) Muy abundante
B) Abundante

8. Hora en la que caza

12. ¿En qué zonas los ha visto? A) Dentro

13. ¿Cuántos estima que caza por año?

14. ¿Ha tenido usted problemas con los mamíferos?

15. Cada cuando come carne de monte A) 2 veces a la semana B) 1 vez a la semana C) 1 vez al mes

D) 1 vez cada 3 meses E) $1 \mathrm{vez}$ al año

16. Cuántos kilos aproximadamente de carne de monte consume 